\title{
feature
}

\section{Standpoint on the priority of TNTs and CNTs as targeted drug delivery systems}

Yasmin Ranjous, Géza Regdon Jr., Klára Pintye-Hódi and Tamás Sovány, t.sovany@pharm.u-szeged.hu

Conventional drug delivery systems have limitations according to their toxicity and poor solubility, bioavailability, stability, and pharmacokinetics (PK). Here, we highlight the importance of functionalized titanate nanotubes (TNTs) as targeted drug delivery systems. We discuss the differences in the physicochemical properties of TNTs and carbon nanotubes (CNTs) and focus on the use of functionalization to improve their characteristics. TNTs are promising materials for drug delivery systems because of their superb properties compared with CNTs, such as their processability, wettability, and biocompatibility. Functionalization improves nanoparticles (NPs) via their surface modification and enables them to achieve the targeted therapy.

\section{Introduction}

Conventional drugs often have poor solubility, PK, biopharmaceutical properties, and stability or cause toxicity [1]. By contrast, nanotechnologybased drug delivery systems can improve the solubility, absorption, permeation, retention time, and bioavailability of drug molecules in target tissues, as well as improving their stability and, therefore, enhancing the shelf-life and acceptability of drugs by increasing either their uptake efficacy or patient compliance [2].

Nanosized delivery systems can be internalized by cells more effectively compared with micro-sized particles. In addition, NPs can be formulated in various shapes, sizes, and compositions, and can be modified physicochemically and functionally to obtain specific properties depending on the requirements of both the drug molecule and the targeted organ [1]. Nanotubes have an ideal inner diameter of 5-6 $\mathrm{nm}$ for loading with large biological molecules, with a surface area five times higher than that of other NPs. Furthermore, cell internalization is higher in the case of tubular NPs compared with their spherical counterparts (H.P. Kulkarni, PhD thesis, University of North Carolina at Chapel Hill, 2008).

The first nanotubes to be discovered were CNTs. The first synthesis method was described by Lijima in 1991, whereas TNTs were first synthesized by Hoyer via template-assisted synthesis in 1996 (reviewed in Ref. [3]). Nevertheless, over the past decades, numerous synthesis routes with various advantages and disadvantages have been developed (Tables 1 and 2).

\section{Structure and classification}

Although both CNTs and TNTs have a tubular structure, there are general differences in their structure. CNTs are allotropes of carbon made from graphene/graphite and are rolled up into concentric cylinders with various wall numbers, on which their classification is based.

Single-walled CNTs (SWNTs) have a diameter of $1 \mathrm{~nm}$ and length up to centimeters, prepared by rolling a single graphene sheet to form a cylinder. The conducting properties of SWNTs depend on the wrapping nature [10], which is represented by chiral vectors $(n, m)$. A zigzag structure is obtained when $m=0$, an armchair is obtained when $n=m$, and a chiral structure is obtained when $m$ lies between the zigzag and the armchair structure values.

Although double-walled CNTs (DWNTs) generally have the same morphology and properties as SWNTs [11], they also exhibit several advantages, such as significantly improved resistance to chemicals, the same thermal and electrical stability as multiwalled CNTs (MWNTs), but the same flexibility as SWNTs [12].

MWNTs have a diameter from $2 \mathrm{~nm}$ to $100 \mathrm{~nm}$ and a length of tens of microns. They have two 
TABLE 1

Comparison of CNT preparation methods

\begin{tabular}{|c|c|c|c|c|}
\hline Method & Product & Advantages & Disadvantages & Refs \\
\hline Arc discharge & $\begin{array}{l}\text { SWNTs } 0.6-1.4 \mathrm{~nm} \text { in diameter or; } \\
\text { MWNTs with } 1-3 \mathrm{~nm} \text { inner and } \\
10 \mathrm{~nm} \text { outer diameter }\end{array}$ & $\begin{array}{l}\text { Upscalable for volume production; } \\
\text { nanotube diameter distribution can } \\
\text { vary; yield up to } 90 \%\end{array}$ & $\begin{array}{l}\text { Solid graphite source required; } \\
\text { requires high temperature; SWNTs } \\
\text { only obtained with use of metal }\end{array}$ & [4] \\
\hline Laser ablation & $\begin{array}{l}\text { SWNTs } 1-2 \mathrm{~nm} \text { in diameter and 5- } \\
20 \mu \mathrm{m} \text { long, or fullerenes }\end{array}$ & $\begin{array}{l}\text { High-quality nanotubes; yield up to } \\
70 \%\end{array}$ & $\begin{array}{l}\text { Solid graphite source required; not } \\
\text { suitable for manufacture of MWNTs } \\
\text { because of short length }\end{array}$ & {$[5]$} \\
\hline Chemical vapor deposition (CVD) & $\begin{array}{l}\text { SWNTs } 0.6-4 \mathrm{~nm} \text { in diameter or } \\
\text { MWNTs } 10-240 \mathrm{~nm} \text { in diameter }\end{array}$ & $\begin{array}{l}\text { Distinguished configuration and } \\
\text { positional control }\end{array}$ & $\begin{array}{l}\text { Two-step method; typical yield is } \\
30 \% \text {; often riddled with defects }\end{array}$ & [6] \\
\hline Plasma-enhanced CVD & SWNTs or MWNTs & No solid graphite source required & Complicated process & [6] \\
\hline Alcohol catalytic CVD & SWNTs $1 \mathrm{~nm}$ in diameter & $\begin{array}{l}\text { SWNTs produced on large scale and } \\
\text { at low cost }\end{array}$ & $\begin{array}{l}\text { Obstacles in creating high-purity } \\
\text { SWNTs }\end{array}$ & [6] \\
\hline Hydrothermal Methods & $\begin{array}{l}\text { MWNTs with } 10-100 \mathrm{~nm} \text { inner and } \\
50-150 \mathrm{~nm} \text { outer diameter } \\
\text { nanorods, nanowires, nanobelts } \\
\text { and nano-onions }\end{array}$ & $\begin{array}{l}\text { Starting materials stable at ambient } \\
\text { temperature; low temperature } \\
\left(150-180^{\circ} \mathrm{C}\right) \text { required; no } \\
\text { hydrocarbon or carrier gas required }\end{array}$ & & [7] \\
\hline
\end{tabular}

TABLE 2

\begin{tabular}{|c|c|c|c|}
\hline Method & Advantages & Disadvantages & Refs \\
\hline Electrochemical treatment & $\begin{array}{l}\text { Self-organized TNT layers with large }(100 \mathrm{~nm}) \\
\text { diameter; suitable for surface modification of } \mathrm{Ti} \\
\text { implants }\end{array}$ & $\begin{array}{l}\text { Length varies }(2-101 \mu \mathrm{m}) \text {; not suitable for many } \\
\text { biomedical applications because of size and potential } \\
\text { clearance by reticuloendothelial system }\end{array}$ & {$[8]$} \\
\hline Template-assisted synthesis & $\begin{array}{l}\text { Variable }(50-400 \mathrm{~nm}) \text { diameter based on template } \\
\text { pore size }\end{array}$ & & [9] \\
\hline Hydrothermal treatment & $\begin{array}{l}\text { Small }(5-10 \mathrm{~nm}) \text { diameter and } 100-1000 \mathrm{~nm} \text { length; } \\
\text { variable dimensions, porosity and specific surface } \\
\text { depending on temperature, } \mathrm{NaOH} \text { concentration, } \\
\text { sonication and acidic post-treatment }\end{array}$ & $\begin{array}{l}\text { Strongly agglomerated TNTs, which need to be } \\
\text { dispersed before bioapplication; nanosheets result as } \\
\text { byproducts ( } 10 \% \text { of batch) }\end{array}$ & {$[8]$} \\
\hline
\end{tabular}

structural models: the 'Russian Doll' model, when graphite sheets are ordered in concentric cylinders (Fig. 1), and the 'Parchment' model [11], when a single sheet of graphite is rolled in around itself. The layers have different chiralities with inconsiderable interlayer electronic coupling, and can shift randomly between metallic and semiconducting varieties. The main advantage of MWNTs is that their stiffness is higher than that of SWNTs, especially during compression [12]. The length-to-diameter ratio of MWNTs is $>1000000$ given that they are nanometers in diameter and several millimeters in length [3].

By contrast, TNTs are rolled up into a spiral (Fig. 1). with an inner cavity of $4 \mathrm{~nm}$ and have an amorphous or crystalline structure depending on the specific electrochemical parameters [8]. The TNTs obtained after anodization are amorphous and not photoactive, whereas high temperature annealing converts amorphous TNTs into a crystalline form (anatase or rutile) and, hence, broadens their application range. TNTs are classified according to the synthesis parameters used to prepare TNTs, such as with template-assisted synthesis, hydrothermal treatments, or electrochemical treatments (H.P.
Kulkarni, PhD thesis, University of North Carolina at Chapel Hill, 2008), which cause variations in their physical features (e.g., length, and inner diameter and outer diameter distributions).

\section{Comparison of the physicochemical properties}

CNTs have highly hydrophobic surfaces because they preserve the apolar characteristics of native graphene/graphite nanosheets and are insoluble in aqueous solutions [13], where the surface charge of CNTs is a function of the $\mathrm{pH}$ of the solution [14]. However, their solubility can be enhanced by functionalization [12], which can also facilitate their movement in the body and reduce both the blockage of body organ

pathways and toxicity, partially by hindering the accumulation of highly apolar molecules in tissue. Nevertheless, the grade of toxicity (in vivo and in vitro) is determined by diverse factors, such as size, shape, purity, surface chemistry, and the existence of transition metal catalysts. Furthermore, it appears that the effect of CNTs on organs is related to the administration route used [15]. Intravenous, oral, and dermal administration of CNTs can cause only mild symptoms, whereas inhalation can result in severe inflammation and toxicity to the respiratory system. By contrast, another study reported that no significant lung inflammation or tissue damage was observed following direct inhalation of CNTs.

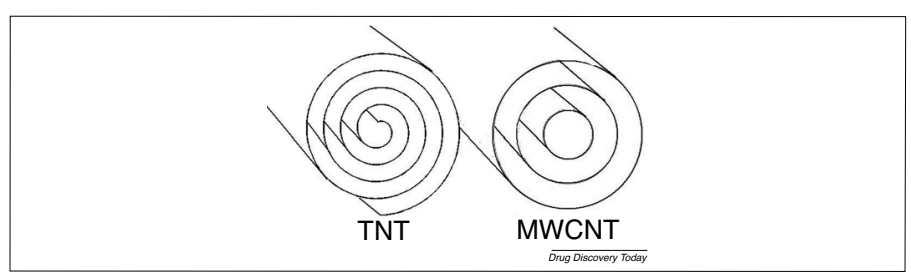

FIGURE 1

Schematic representation of the structural differences between titanate nanotubes (TNTs) and multiwalled carbon nanotubes (MWCNTs). 
By contrast, TNTs display strong hydrophilicity because of their partially hydroxylated surface, which causes a negative $\zeta$-potential (after washing until $\mathrm{pH}=6$ ) that, when combined with hydrogen bonds, causes superior wettability [16] but often leads to the agglomeration of the particles, especially in dry forms [8]. Their hydrophilicity is also supported by the capillary effect, resulting in the quick penetration of water droplets into the tube pores, and by their crystallinity, given that the amorphous, mixed crystalline phase shows high polarity because of the O-Ti-O bonds and to the extensive presence of hydroxyl groups on the TNT surface. Furthermore, the structure of TNTs also influences the contact angle, which decreases with increases in both tube and pore diameters and with increasing anodization voltage or thermal treatment up to $450{ }^{\circ} \mathrm{C}$; however, beyond $450{ }^{\circ} \mathrm{C}$, their hydrophilicity decreases because of the detachment of hydroxyl groups from the surface [17]. The high surface energy and polarity causes good wettability and, hence, improved cell adhesion. Therefore, TNTs showed extremely good biocompatibility. Bone cell adhesion and differentiation were improved by the use of TNT-covered implants and were proven to be better than those with a pure Ti surface. TNTs were also nontoxic when internalized by cells [18-20]; thus, they appear to have good applicability for therapeutic use in the clinic [21].

Despite their different surface characteristics, CNTs and TNTs exhibit considerable similarities regarding their impressive mechanical, electrical, and optical properties. Nanotubular structures usually have good mechanical properties. In CNTs, the covalent bonds between carbon atoms lead to high tensile strength (up to 63 $\mathrm{GPa}$ ) and Young's modulus of elasticity (1-1.8 TPa depending on the diameter and the chirality of the tube) [3]. Therefore, SWNTs are stronger than steel by 10 to 100 times per unit weight. By contrast, MWNTs have lower Young's modulus values than SWNTs because stress is only supported by the outer graphite shelf on account of weak intertube cohesion. Similarly, TNTs exhibit high, but one grade lower Young's modulus $(230 \mathrm{GPa})$ and tensile strength (680 MPa) compared with SWNTs. Nevertheless, these values still reflect impressive mechanical properties, supported by the results of Sipos et al., who reported that TNTs and their composites formed with various drugs showed supreme flowability, compressibility, and compactibility compared with crystalline APIs, thus proving their superior processability [22-24]. In terms of their electrical behavior, CNTs display semiconducting or metallic resistance, capacitance, and inductance properties because of their electronic structure and symmetry of graphene [12]. SWNTs can be either semiconducting or metallic, whereas MWNTs are semiconducting. The electrical conductivity of self-organized TNTs is based on their crystalline structure and is tunable with the annealing temperature, because when the amorphous material converts into anatase at $300^{\circ} \mathrm{C}$, it results in significantly higher conductivity, whereas the conversion of anatase into the more resistive rutile above $500{ }^{\circ} \mathrm{C}$ reduces the conductivity [25]. In terms of their optical properties, both CNTs and TNTs show optical absorbance: the absorbance of CNTs is in near-infrared (NIR) zone [12], whereas TNTs display wider photo absorption properties, although not as good as $\mathrm{TiO}_{2}$ NPs. However, when rare earth ions $\left(\mathrm{Pr}^{31}, \mathrm{Er}^{31}, \mathrm{Nd}^{31}\right.$, and $\left.\mathrm{Yb}^{31}\right)$ were intercalated into TNTs, higher photoluminescence emission was observed compared with pristine Na-TNTs [26]. Overall, these remarkable properties make CNTs and TNTs an ideal target for a range of diagnostic, biomedical, or pharmaceutical applications.

\section{Applications}

The high binding capacity and unique physicochemical, especially electrical properties of nanotubes can be well utilized in specific molecule recognition and other diagnostic applications. CNTs can be used as biosensors to diagnose diseases, record the pulse and temperature of a patient, and measure blood glucose, or other biomolecules, such as $\mathrm{H}_{2} \mathrm{O}_{2}$, organophosphate pesticides, or cancer markers, in diagnosis and treatment [12,27-29]. In addition, their good biocompatibility and mechanical properties also make nanotubular structures suitable for tissue-engineering applications.

CNTs can improve the mechanical strength of implanted catheters and, hence, reduce

thrombus formation in cardiovascular surgeries [12]. CNT-coated polyurethane has high interconnected porosity, bioactivity, and nanostructured surface topography. Thus, CNTs can be used as bioactive scaffolds in bone tissue engineering and provide new properties, such as electrical conductivity, to these scaffolds [30], or, when filled with calcium, they can be used directly as a bone substitute, with improved mechanical properties because of their high tensile strength [3]. Consequently, they can help in directing cell growth [12]. Correspondingly, TNT coatings on scaffolds reinforce cell growth on the biodegradable photopolymer scaffolds [31] and also promote bone formation by hastening osteoblast growth by $300-400 \%$ compared with non-anodized Ti surfaces [32]. This effect was further improved when TNTs were coated with biocompatible polymer films comprising chitosan and poly(lactic-co-glycolic acid), when superior osteoblast adhesion and cell proliferation were achieved, compared with uncoated TNTs [33].

Given their unique characteristics, such as their hollow monolithic structure, nanoneedle shape, considerable molecule-binding capacity and versatile binding mechanisms, nanotubes are also ideal carriers in other biomedical and pharmaceutical applications. Two different methods exist for binding: wrapping, when drugs and biological molecules are attached to the surface through functional groups; and filling, when drugs and biological molecules are loaded inside CNTs [34].

CNTs display immunogenicity and devised antibody responses linked to viral protein VP1 of foot-and-mouth disease virus (FMDV), which could be utilized for the stimulation of the immune system [3]. The high RNA binding and internalization capacity also make CNTs suitable for cytoplasm or cell core targeting and valuable as vectors to transfer genes and drugs into cells to cure cancer and various genetic disorders [35]. However, SWNTs are more useful compared with MWNTs because of their 1D structure, efficient drug-loading capacity, and large surface area [36]. CNTs conjugated to small interfering (si)RNA molecules were successful in silencing the expression of CD4 cell surface receptors and CXCR4 co-receptors, thus inhibiting the infection of T cells by HIV [37]. Drugembedded CNTs can also be utilized to kill viruses in viral ulcers without antibody production against the drug, because viruses present no intrinsic immunogenicity for CNTs [38]. CNTs can carry streptavidin and cytochrome $\mathrm{C}$ into the cell cytoplasm via the endocytosis pathway [12] and showed high selectivity to kill cancer cells after internalization, achieved by hyperthermia because of their thermal conductivity [39]. However, MWCTs are more suitable than are SWCTs for thermal cancer treatment given that MWNTs absorb NIR radiation faster than do SWNTs [40].

Nevertheless, CNTs can be applied for drug delivery and targeting without external stimulation because the SWCNT-anticancer drug complex increases blood circulation time, enhancing permeability and the retention effect by tumor cells [41], as shown by the successful delivery of amphotericin B [42], the successful delivery and retention of polyphosphazene platinum to the brain [43], the successful oral administration of erythropoietin (EPO) [43] and the slow release of cisplatin in an aqueous 
environment to terminate the growth of human lung cancer cells [44].

Based on their physicochemical properties, TNTs offer fewer opportunities to attach drugs or other molecules; however, based on their unique properties, such as biocompatibility, mechanical strength, and chemical resistivity, they are proposed to be ideal materials for the development of various medical implants and devices. Thus, TNTs have so far been applied mainly in dentistry, orthopedics, and cardiovascular surgery [45].

\section{Functionalization of TNTs and CNTs}

Functionalization is the attaching of appropriate molecules to the nanostructure surface to render them soluble in water, reduce toxicity, increase biocompatibility [46], achieve targeted drug delivery, obtain selective binding to the desired epitope, achieve controlled drug release, facilitate cellular internalization, enhance biodistribution, and improve biofluid circulation. Many types of functionalization molecule have been used, such as polyethylene glycol (PEG), polyvinylpyrrolidone (PVP), cellulose, polypeptides, dextran, and silica [2].

CNTs can be functionalized covalently or noncovalently on the tips and side walls, although CNT tips have a higher functionalization affinity compared with the side walls [46]. Noncovalent functionalization, including Van der Waals interactions, $\pi-\pi$ interactions, and hydrophobic interactions, causes minimal damage to the CNT surface and maintains the aromatic structure and, consequently, the electronic characteristics of CNTs. However, the disadvantage is that this kind of functionalization is not appropriate for targeted drug delivery applications because of the weak forces formed [47]. By contrast, covalent functionalization of CNTs can be achieved via oxidizing them by strong acids, such as nitric and sulfuric acids [48]. Hence, the forming of carboxylic acid groups because of the high negative charge increases the hydrophilicity, water solubility, and biocompatibility of CNTs [49]. By contrast, the disadvantage is that covalent functionalization damages CNT side walls and, thus, CNTs cannot be used in some applications, such as imaging [37]. Nevertheless, the presence of carboxylic and other oxygencontaining groups on the surface of CNTs also allows the covalent attachment of functional molecules [50]. The covalent surface functionalization of CNTs with amine-terminated PEG stabilizes CNT dispersions in various media and reduces deleterious effects on cultured cells [51], and oxidation debris (i.e., the breaking CNTs during oxidation or oxidizing carbonaceous nontubular structures in pristine CNT samples).

Similarly, the surface characteristics, such as the negative charge at physiological $\mathrm{pH}$ caused by the presence of hydroxyl groups on their surface above their isoelectric point (pH 3.7), enable TNTs to react with a variety of functional molecules [52]. The functionalization of TNTs improves their stability for vectorization applications and enables them to carry therapeutic molecules [53]. Tables 3 and 4 detail methods for the functionalization of CNTs and TNTs, respectively.

TABLE 3

\section{Functionalization possibilities of CNTs}

\begin{tabular}{|c|c|c|}
\hline Reagent(s) & Aim of functionalization/grafting & Refs \\
\hline Nitric acid $\left(\mathrm{HNO}_{3}\right)$ & Carboxylic groups covered MWNTs; increase solubility & [54] \\
\hline $\mathrm{NH}_{2}\left(\mathrm{CH}_{2} \mathrm{CH}_{2} \mathrm{O}\right)_{2}-\mathrm{CH}_{2} \mathrm{CH}_{2} \mathrm{NH}_{2}$ & $\begin{array}{l}\mathrm{NH}_{2} \text { covering of MWNTs; increase solubility; decrease aggregation; decrease cytotoxic } \\
\text { effects }\end{array}$ & {$[55]$} \\
\hline Second-generation poly (amidoamine) dendrimer & Increase surface binding ability of DNA probe by supplying large number of amino groups & {$[56]$} \\
\hline
\end{tabular}

(G $\mathrm{G}_{2}$-PAMAM)

Folate moiety

Phospholipid-PEG2000- $\mathrm{NH}_{2}$ $\mathrm{HNO}_{3}$ and salicylaldehyde

$\mathrm{HNO}_{3}$ and $\mathrm{H}_{2} \mathrm{SO}_{4}$ mixture; 1-(3-dimethylaminopropyl)-3ethylcarbodiimide hydrochloride; $N$-hydroxysuccinimide;

P-glycoprotein antibody
Selective destruction of cancer cells labeled with folate receptor tumor markers; NIR-triggered cell death without harming receptor-free normal cells

Photothermal cancer treatment in mice by NIR irradiation

Reduce reaction step number and reaction time

Specific recognition of multidrug-resistant human leukemia cells (K562R)
[39]

TABLE 4

\section{Functionalization possibilities of TNTs}

\begin{tabular}{|c|c|c|}
\hline Reagent(s) & Aim of functionalization/grafting & Res \\
\hline $\begin{array}{l}\text { Dopamine; Tris buffer; bone } \\
\text { morphogenetic protein } 2\left(\mathrm{BMP}_{2}\right)\end{array}$ & Enhance bone osseointegration & {$[58]$} \\
\hline $\begin{array}{l}\text { 3-isocyanatopropyltriethoxy; PEG; } \\
\text { polyethylene imine (PEI) }\end{array}$ & Enhance TNT dispersion in water and reactivity & {$[53]$} \\
\hline Allyltriethoxysilane; propyltriethoxysilane & Form stable suspensions in tetrahydrofuran (THF) & [59] \\
\hline Antimicrobial peptides (HHC-36) & Prevent formation of biofilms (based on bactericide and bacteriostatic effect) & {$[60]$} \\
\hline $\begin{array}{l}\text { 3-aminopropyltriethoxysilane; RGD } \\
\text { peptide }\end{array}$ & Promote initial attachment and proliferation of human mesenchymal stem cells (hMSCs) & {$[61]$} \\
\hline KRSR & Increase osteogenic differentiation and pre-osteoblast adhesion and spread on TNT surface & {$[62]$} \\
\hline $\begin{array}{l}\mathrm{N}, \mathrm{N} \text {-carbonyl diimidazole; } 11 \text {-hydroxy- } \\
\text { undecylphosphonic acid; EGF and } \mathrm{BMP}_{2} \\
\text { growth factors }\end{array}$ & Increasing number and activity of MSCs & {$[63]$} \\
\hline Gelatin-stabilized gold NPs & Improve MC3T3-E1 osteoblast cell adhesion and propagation (achieved) & [64] \\
\hline Chitosan & Achieve sustained release of loaded drug (selenium or quercetin) from TNTs & {$[65,66]$} \\
\hline
\end{tabular}




\section{Concluding remarks}

Drug delivery devices based on nanotubular structures are ideal for modern theranostic applications because of their advantageous properties. However, they can bear the risk of toxicity attributable to their size, surface charge, chemical composition, chemical reactivity, chemical structure, crystal structure, shape, solubility, and degree of agglomeration. Moreover, nanomaterials can cause oxidative stress and damage phagocytosis inside the cells, reduce cell viability, and suppress cell proliferation by producing reactive oxygen species or remaining in the body because of their ability to evade the reticuloendothelial system.

Despite many promising results and numerous advantages, pristine CNTs are insoluble in water and most solvents; thus, they cannot be used immediately in biomedical applications. Furthermore, they bear a considerable risk of toxicity and carcinogenicity because they accumulate in the human body because of their strongly hydrophobic nature and residual metal catalysts, which increases their ability to produce $\mathrm{O}^{2-}$ anions, lipid peroxidation, or physical blockage generated from agglomeration at high doses, given that CNTs also have a strong electrostatic attraction.

By contrast, TNTs have exhibited promising toxicological profiles and good biocompatibility in numerous studies and a vital affinity for bone cell adhesion and differentiation, which allows their use in dentistry, orthopedics, and cardiovascular surgery. Therefore, and as a result of their tubular structure, CNT-similar chemical resistivity, mechanical strength, and electron mobility, TNTs might be promising alternatives for developing medical implants and devices. Nevertheless, despite these advantages, TNTs, especially hydrothermally synthetized free TNTs, are poorly studied in terms of their use in drug delivery applications, possibly because of their hydrophilic nature, which improves their biocompatibility and decreases the risk of adverse effects, but also acts negatively on their absorption and cell internalization properties. Thus, functionalization might be key to improving their applicability, given that the range of possibilities is almost as wide as for CNTs. Noncovalent bindings based on van der Waals forces, hydrogen bonds or $\pi-\pi$ interactions are easily achievable, which maintain the aromatic structure and electronic characteristics; obtaining covalent functionalization with etheror esterification of the free surface - $\mathrm{OH}$ groups is also possible. With the selection of the appropriate functional groups, the surface properties and, therefore, their absorption and internalization capacity could be improved without the considerable elevation of the risk of toxicity. Furthermore, their similar mechanical, electrical, and optical parameters could provide the same level of processability and range for external stimuli-adjusted targeting possibilities as CNTs.

In terms of their low toxicity and advantageous physicochemical properties, the further investigation, use, and application of hydrothermally synthetized TNTs is recommended for the development of new advanced drug delivery systems.

\section{References}

1 Suri, S.S. et al. (2007) Nanotechnology-based drug delivery systems. J. Occup. Med. Toxicol. 2, 16

2 Raliya, R. et al. (2016) Perspective on nanoparticle technology for biomedical use. Curr. Pharma. Des. 22, 2481-2490

3 Mahajan, D. (2017) Carbon nanotubes: a review on synthesis, electrical and mechanical properties and applications. Asian J. Appl. Sci. Technol. 1, 15-20

4 Ebbesen, T. and Ajayan, P. (1992) Large-scale synthesis of carbon nanotubes. Nature 358, 220

5 Sivaram, A. (2004) Laser ablation process for singlewalled carbon nanotube production. J. Nanosci. Nanotechnol. 4, 317-325

6 Khurshed, A. et al. (2016) Synthesis of carbon nanotubes by catalytic chemical vapour deposition: a review on carbon sources, catalysts and substrates. Mater. Sci. Semicond. Process. 41, 67-82

7 Gogotsi, Y. and Libera, J.A. (2000) Hydrothermal synthesis of multiwall carbon nanotubes. J. Mater. Res. $15,2591-2594$

8 Boudon, J. et al. (2014) Titanate nanotubes as a versatile platform for nanomedicine. In Nanomedicine (Seifalian, A., ed.), pp. 403-429, One Central Press, Altricham, UK

9 Rørvik, P.M. et al. (2009) Template-assisted synthesis of PbTiO3 nanotubes. J. Eur. Ceram. Soc. 29, 2575-2579

10 Odom, T.W. et al. (1998) Atomic structure and electronic properties of single-walled carbon nanotubes. Nature 391, 62-64

11 Mamedov, A.A. et al. (2002) Molecular design of strong single-wall carbon nanotube/polyelectrolyte multilayer composites. Nat. Mater. 1, 190-194

12 Kumar, S.P. et al. (2012) Pharmaceutical application of carbon nanotube-mediated drug delivery system. Int. J. Pharm. Sci. Nanotechnol. 5, 1685-1696

13 Liu, Z. et al. (2009) Carbon nanotubes in biology and medicine: in vitro and in vivo detection, imaging and drug delivery. Nano Res. 2, 85-120

14 Dezfoli, A.R.A. et al. (2013) Structural properties of water around uncharged and charged carbon nanotubes. Kor. J. Chem. Eng. 30, 693-699

15 Smart, S.K. et al. (2006) The biocompatibility of carbon nanotubes. Carbon 44, 1034-1047

16 Wang, F. et al. (2013) Bioinspired micro/nano fabrication on dental implant-bone interface. Appl. Surf. Sci. 265, 480-488

17 Indira, K. et al. (2015) A review on $\mathrm{TiO} 2$ nanotubes: influence of anodization parameters, formation mechanism, properties, corrosion behavior, and biomedical applications. J. Bio. Tribo. Corr. 1, 28
18 Papa, A.-L. et al. (2012) Titanate nanotubes: towards a novel and safer nanovector for cardiomyocytes. Nanotoxicology 7, 1131-1142

19 Mirjolet, C. et al. (2013) The radiosensitization effect of titanate nanotubes as a new tool in radiation therapy for glioblastoma: a proof-of-concept. Radiother. Oncol. 108, 136-142

20 Fenyvesi, F. et al. (2014) Investigation of the cytotoxic effects of titanate nanotubes on Caco-2 cells. AAPS PharmSciTech 15, 858-861

21 Wang, Q. et al. (2016) TiO2 nanotube platforms for smart drug delivery: a review. Int. J. Nanomedicine 11, 4819-4834

22 Matsuno, R. et al. (2004) Polystyrene-and poly (3vinylpyridine)-grafted magnetite nanoparticles prepared through surface-initiated nitroxide-mediated radical polymerization. Macromolecules 37, 2203-2209

23 Sipos, B. et al. (2017) Comparative study on the rheological properties and tablettability of various APIs and their composites with titanate nanotubes. Powder Technol. 321, 419-427

24 Sipos, B. et al. (2018) Investigation of the compressibility and compactibility of titanate nanotube-API composites. Materials 11, 2582

25 Tighineanu, A. et al. (2010) Conductivity of TiO2 nanotubes: Influence of annealing time and temperature. Chem. Phys. Lett. 494, 260-263

26 Marques, T.M.F. et al. (2017) Photoluminescence enhancement of titanate nanotubes by insertion of rare earth ions in their interlayer spaces. J. Nanomater. 2017, 3809807

27 Bandaru, P.R. (2007) Electrical properties and applications of carbon nanotube structures. J. Nanosci. Nanotechnol. 7, 1239-1267

28 Zhang, M. and Gorski, W. (2005) Electrochemical sensing platform based on the carbon nanotubes/ redox mediators-biopolymer system. ACC Chem. Res. 127, 2058-2059

29 Liu, G. and Lin, Y. (2006) Biosensor based on selfassembling acetylcholinesterase on carbon nanotubes for flow injection/amperometric detection of organophosphate pesticides and nerve agents. Anal. Chem. 78, 835-843

30 Harrison, B.S. and Atala, A. (2007) Carbon nanotube applications for tissue engineering. Biomaterials 28, 344-353

31 Minagar, S. et al. (2013) Cell response of anodized nanotubes on titanium and titanium alloys. J. Biomed. Mater. Res. A 101, 2726-2739

32 Yamamoto, A. et al. (1998) A new technique for direct measurement of the shear force necessary to detach a cell from a material. Biomaterials 19, 871-879

33 Gulati, K. et al. (2012) Biocompatible polymer coating of titania nanotube arrays for improved drug elution and osteoblast adhesion. Acta Biomater. 8, 449-456

34 Sahoo, N.G. et al. (2011) Functionalized carbon nanomaterials as nanocarriers for loading and delivery of a poorly water-soluble anticancer drug: a comparative study. Chem. Commun. 47, 5235-5237

35 Kam, N.W.S. et al. (2005) Functionalization of carbon nanotubes via cleavable disulfide bonds for efficient intracellular delivery of siRNA and potent gene silencing. J. Am. Chem. Soc. 127, 12492-12493

36 Madani, S.Y. et al. (2011) A new era of cancer treatment: carbon nanotubes as drug delivery tools. Int. J. Nanomedicine 6, 2963-2979

37 Liu, Z. et al. (2009) Preparation of carbon nanotube bioconjugates for biomedical applications. Nat. Protoc 4, 1372-1381 
38 Pantarotto, D. et al. (2003) Immunization with peptidefunctionalized carbon nanotubes enhances virusspecific neutralizing antibody responses. Chem. Biol. 10, 961-966

39 Kam, N.W.S. et al. (2005) Carbon nanotubes as multifunctional biological transporters and nearinfrared agents for selective cancer cell destruction. Proc. Natl. Acad. Sci. U. S. A. 102, 11600-11605

40 Hirsch, L.R. et al. (2003) Nanoshell-mediated nearinfrared thermal therapy of tumors under magnetic resonance guidance. Proc. Natl. Acad. Sci. U. S. A. 100, 13549-13554

41 Liu, Z. et al. (2008) Drug delivery with carbon nanotubes for in vivo cancer treatment. Cancer Res. 68 6652-6660

42 Barroug, A. and Glimcher, M.J. (2002) Hydroxyapatite crystals as a local delivery system for cisplatin: adsorption and release of cisplatin in vitro. J. Orthop. Res. 20, 274-280

43 Pai, P. et al. (2006) Pharmaceutical applications of carbon tubes and nanohorns. Pharm. Res. 1, 11-15

44 Ajima, K. et al. (2005) Carbon nanohorns as anticancer drug carriers. Mol. Pharm. 2, 475-480

45 Rahman, Z.U. et al. (2016) Electrochemical \& osteoblast adhesion study of engineered TiO 2 nanotubular surfaces on titanium alloys. Mater. Sci. Eng. C 58, 160-168

46 Prato, M. et al. (2007) Functionalized carbon nanotubes in drug design and discovery. ACC Chem. Res. 41, 60-68

47 Liu, Z. et al. (2007) Supramolecular chemistry on watersoluble carbon nanotubes for drug loading and delivery. ACS Nano. 1, 50-56

48 Klumpp, C. et al. (2006) Functionalized carbon nanotubes as emerging nanovectors for the delivery of therapeutics. Biochim. Biophys. Acta Biomembr. 1758, 404-412

49 Nagasawa, S. et al. (2000) Effect of oxidation on singlewall carbon nanotubes. Chem. Phys. Lett. 328, 374-380

50 Wang, Y. et al. (2005) Microwave-induced rapid chemical functionalization of single-walled carbon nanotubes. Carbon 43, 1015-1020
51 Moon, H.K. et al. (2009) In vivo near-infrared mediated tumor destruction by photothermal effect of carbon nanotubes. ACS Nano 3, 3707-3713

52 Papa, A.-L. et al. (2011) Synthesis of titanate nanotubes directly coated with USPIO in hydrothermal conditions: a new detectable nanocarrier. J. Phys. Chem. C 115, 19012-19017

53 Papa, A.-L. et al. (2015) Dispersion of titanate nanotubes for nanomedicine: comparison of PEI and PEG nanohybrids. Dalton Trans. 44, 739-746

54 Chen, C.-C. et al. (2007) Modification of multiwalled carbon nanotubes by microwave digestion method as electrocatalyst supports for direct methanol fuel cell applications. Electrochem. Comm. 9, 159-163

55 Coccini, T. et al. (2010) Effects of water-soluble functionalized multi-walled carbon nanotubes examined by different cytotoxicity methods in human astrocyte D384 and lung A549 cells. Toxicology 269, 41-53

56 Zhu, N. et al. (2010) Sensitive impedimetric DNA biosensor with poly (amidoamine) dendrimer covalently attached onto carbon nanotube electronic transducers as the tether for surface confinement of probe DNA. Biosens. Bioelectron. 25, 1498-1503

57 Li, R. et al. (2010) P-glycoprotein antibody functionalized carbon nanotube overcomes the multidrug resistance of human leukemia cells. ACS Nano. 4, 1399-1408

58 Lai, M. et al. (2011) Surface functionalization of TiO2 nanotubes with bone morphogenetic protein 2 and its synergistic effect on the differentiation of mesenchymal stem cells. Biomacromolecules 12, 1097-1105

59 Byrne, M.T. et al. (2007) Chemical functionalisation of titania nanotubes and their utilisation for the fabrication of reinforced polystyrene composites. J. Mater. Chem. 17, 2351-2358

60 Kazemzadeh-Narbat, M. et al. (2013) Multilayered coating on titanium for controlled release of antimicrobial peptides for the prevention of implantassociated infections. Biomaterials 34, 5969-5977

61 Oh, S. et al. (2013) Effect of RGD peptide-coated TiO 2 nanotubes on the attachment, proliferation, and functionality of bone-related cells. J. Nanomater. 2013 965864

62 Oliveira, W.F. et al. (2017) Functionalization of titanium dioxide nanotubes with biomolecules for biomedical applications. Mater. Sci. Eng. C 81, 597-606

63 Bauer, S. et al. (2011) Covalent functionalization of TiO 2 nanotube arrays with EGF and BMP-2 for modified behavior towards mesenchymal stem cells. Integr. Biol. 3, 927-936

64 Neupane, M.P. et al. (2011) Titania nanotubes supported gelatin stabilized gold nanoparticles for medical implants. J. Mater. Chem. 21, 12078-12082

65 Chen, X. et al. (2013) Fabrication of selenium-deposited and chitosan-coated titania nanotubes with anticancer and antibacterial properties. Coll. Surf. B: Biointerfaces 103, 149-157

66 Mohan, L. et al. (2016) Drug release characteristics of quercetin-loaded $\mathrm{TiO}_{2}$ nanotubes coated with chitosan. Int. J. Biol. Macromolecul. 93, 1633-1638

\section{Yasmin Ranjous \\ Géza RegdonJr. \\ Klára Pintye-Hódi \\ Tamás Sovány*}

University of Szeged, Institute of Pharmaceutical Technology and Regulatory Affairs, H-6720, Eötvös u. 6, Szeged, Hungary

${ }^{*}$ Corresponding author. 